\title{
Publisher Correction: Diverse populations of local interneurons integrate into the Drosophila adult olfactory circuit
}

\author{
Nan-Fu Liou (1) ', Shih-Han Lin', Ying-Jun Chen', Kuo-Ting Tsai', Chi-Jen Yang (1) ', Tzi-Yang Lin ${ }^{1,4}$, Ting-Han Wu', \\ Hsin-Ju Lin', Yuh-Tarng Chen', Daryl M. Gohl (1) ${ }^{2,5}$, Marion Silies ${ }^{2,6}$ \& Ya-Hui Chou (i) ${ }^{1,3}$
}

Correction to: Nature Communications 9, 2232; https://doi.org/10.1038/s41467-018-04675-x; published online 8 June 2018

The original version of this Article contained errors in Figs. 4 and 6. In Fig. 4, panel a, text labels UAS-FLP and LexAop2>stop $>$ myr:: smGdP-HA were shifted upwards during typesetting of the figure, and in Fig. 6, panel h, the number 15 was incorrectly placed on the heat map scale. These have now been corrected in both the PDF and HTML versions of the Article.

Published online: 06 November 2018

\begin{abstract}
(c) (i) Open Access This article is licensed under a Creative Commons Attribution 4.0 International License, which permits use, sharing, adaptation, distribution and reproduction in any medium or format, as long as you give appropriate credit to the original author(s) and the source, provide a link to the Creative Commons license, and indicate if changes were made. The images or other third party material in this article are included in the article's Creative Commons license, unless indicated otherwise in a credit line to the material. If material is not included in the article's Creative Commons license and your intended use is not permitted by statutory regulation or exceeds the permitted use, you will need to obtain permission directly from the copyright holder. To view a copy of this license, visit http://creativecommons.org/licenses/by/4.0/.
\end{abstract}

(๑) The Author(s) 2018

\footnotetext{
${ }^{1}$ Institute of Cellular and Organismic Biology, Academia Sinica, Taipei 11529, Taiwan. ${ }^{2}$ Department of Neurobiology, Stanford University, Stanford, CA 94305, USA. ${ }^{3}$ Neuroscience Program of Academia Sinica, Academia Sinica, Taipei 11529, Taiwan. ${ }^{4}$ Present address: Research Institute of Molecular Pathology (IMP), Vienna Biocenter, Campus-Vienna-Biocenter 1, 1030 Vienna, Austria. ${ }^{5}$ Present address: University of Minnesota Genomics Center, 1-210 CCRB, 2231 6th Street SE, Minneapolis, MN 55455, USA. ${ }^{6}$ Present address: European Neuroscience Institute, University Medical Center Göttingen, Grisebachstr. 5, 37077 Göttingen, Germany. These authors contributed equally: Nan-Fu Liou, Shih-Han Lin, Ying-Jun Chen. The original article can be found online at https://doi.org/10.1038/s41467-018-04675-x. Correspondence and requests for materials should be addressed to

Y.-H.C. (email: yhchou@gate.sinica.edu.tw)
} 